\title{
LA EFICIENCIA EN EL DISEÑO ESTRUCTURAL. UN EJERCICIO DE APLICACIÓN EN CONSTRUCCIONES DE MAMPOSTERÍA SISMORRESISTENTE.
}

\section{EFFICIENCY IN STRUCTURAL DESIGN. AN APPLICATION EXERCISE IN EARTHQUAKE RESISTANT IN MASONRY CONSTRUCTIONS.}

\author{
Autor Cátedra de Estructuras III, Facultad de Arquitectura, Urbanismo y Diseño, Univ. Nacional de
} Córdoba, Ciudad de Córdoba, Argentina.

Correo electrónico de contacto: Gustavo González: arq-ggg@hotmail.com Gabriela Asís Ferri: arqgabrielaasis@gmail.com

\section{RESUMEN}

Julieta Mansilla: arq.julietamansilla@gmail.com

La irregularidad de la configuración espacial de los edificios incrementa los efectos perjudiciales de la torsión sobre los planos resistentes, aumentando los esfuerzos, y en consecuencia sobredimensionando la estructura. Ante esta problemática, el REGLAMENTO ARGENTINO PARA CONSTRUCCIONES SISMORRESISTENTES, Parte I, para construcciones en general (INPRES CIRSOC 103 - 2013), incorpora criterios cuantitativos que se suman a los criterios cualitativos existentes, que permiten evaluar la irregularidad torsional a partir de un análisis tridimensional.

Durante el proceso de diseño de una obra de arquitectura se genera una síntesis de las condicionantes funcionales, tecnológicas y formales, siendo el mecanismo estructural una de ellas, por lo que deberá ser concebido por el proyectista desde las primeras etapas del proceso creativo.

En el curso de Estructuras III se realiza un ejercicio de diseño con mampostería encadenada estructural sismorresistente, considerando a la eficiencia como un aspecto fundamental de evaluación de la propuesta. Se orienta al estudiante para que diseñe el mecanismo estructural y reconozca las acciones sobre el mismo, y que a partir del manejo de recursos informáticos, genere un modelo tridimensional para su análisis, verificación y reformulación, a fin de lograr un resultado eficiente.

Se detallan experiencias de diseño de alumnos, donde se verifica el criterio y capacidad de evaluar alternativas de mecanismos estructurales, en esta búsqueda de eficiencia estructural.

\section{ABSTRACT}

The irregularity of the spatial configuration of the buildings increases the damaging effects of torsion on the resistant planes, increasing efforts, and consequently oversizing the structure. Given this problem, the ARGENTINE REGULATION FOR SISMORRESISTENT CONSTRUCTIONS, Part I, for constructions in general (INPRES - CIRSOC 103 - 2013), incorporates quantitative criteria that added to the existing qualitative criteria, allow the evaluation of torsional irregularity from a three-dimensional analysis.

During the design process of an architectural work, a synthesis of the functional, technological and formal conditions is generated, as the structural mechanism is one of these conditions, it must be conceived by the designer from the early stages of the creative process. 
Gustavo González; Gabriela Asís Ferri; Julieta Mansilla

In the course of Structures III, a design exercise with seismic-resistant structural chained masonry is carried out, considering efficiency as a fundamental aspect of the evaluation of the proposal. The student is oriented to design the structural mechanism and recognize the actions on it and throug the use of computer resources, generate a three-dimensional model for analysis, verification and reformulation, in order to achieve an efficient result.

Experiences of student design are detailed, where the criteria and ability to evaluate alternatives of structural mechanisms are verified, in this search for structural efficiency.

PALABRAS CLAVE: CONFIGURACION - CRITERIOS ESTRUCTURALES - ESPACIAL MECANISMO - TORSION

KEY WORDS: CONFIGURATION - STRUCTURAL CRITERIA - SPACE - MECHANISM - TORSION.

Artículo RECIBIDO: 19/07/19 | Artículo ACEPTADO: 10/11/19

\section{INTRODUCCIÓN}

Integrantes Cátedra Estructuras III B: Gustavo G. González, Gabriela Asís Ferri, María Julieta Mansilla, Eduardo Wuthrich, Eduardo Rodríguez Cimino, Raquel Fabre, Daniela Gilabert, Horacio Altamirano, Leonel Ghiglione, Mateo Allende Pose, Anabella Cardelino

La irregularidad de la configuración espacial de los edificios, incrementa los efectos perjudiciales de la torsión sobre los planos resistentes, aumentando los esfuerzos, y en consecuencia sobredimensionando la estructura. El Reglamento Argentino para Construcciones Sismorresistentes, Parte I, para construcciones en general y Parte III, para construcciones de mampostería (INPRES CIRSOC 103- año 2013), hace hincapié, en la valoración de la regularidad de las edificaciones, tanto en planta, como en altura, a partir de ciertos parámetros explicitados en los mismos.

Por otro lado, como docentes de Estructuras III, FAUD, UNC, somos conscientes que, el proceso de aprendizaje y transferencia comprende la incorporación de los conceptos, métodos y estrategias, en la formación académica y en el desempeño de la práctica profesional. En la asignatura mencionada, de cuarto nivel, realizamos un ejercicio de diseño con mampostería encadenada estructural sismorresistente, considerando a la eficiencia como un aspecto fundamental de evaluación de la propuesta.

Se busca que el estudiante examine/diseñe el mecanismo estructural y reconozca las acciones sobre el mismo, luego comprenda el comportamiento de la estructura, para modelarla, analizarla y verificarla con recursos informáticos a fin de lograr un producto eficiente, coherente y compatible con la arquitectura que lo sustenta.

\section{METODOLOGÍA}

El trabajo se puede ordenar en 4 etapas claramente identificables:

A. Selección de obras de arquitectura realizadas con mampostería, con pocos planos portantes (menos de 10), donde la eficiencia estructural no fue un parámetro de diseño.

B. Propuesta de un mecanismo estructural coherente con la arquitectura. Predimensionados de elementos estructurales. Elaboración de la planta de estructura más apropiada para la arquitectura. 
C. Análisis, Verificación y Reformulación de la propuesta. Parámetros de control de deformaciones. Dimensionado de los encadenados y confección de maquetas en escala

D. Elaboración de conclusiones y formulación de criterios de diseño.

\section{DESARROLLO}

En primera instancia, los docentes seleccionaron proyectos reconocidos, de reducidas superficies, con pocos planos resistentes verticales de mampostería, se consideraron ubicados en una determinada zona sísmica y se estableció un sitio.

Para el desarrollo del ejercicio, a cada grupo de alumnos se les asignó una alternativa, con la premisa de respetar las cualidades espaciales, formales y arquitectónicas de cada obra. Los estudiantes plantearon, en cada caso, los posibles mecanismos estructurales, aptos para soportar acciones sísmicas y gravitatorias, analizando, primero cualitativamente y luego, cuantitativamente las obras seleccionadas:

\section{Proyecto A / Casa ZAG (Fig. 1)}

Arquitectos: Estudio BaBo /

Ubicación: Balneario Zagarzazu, Uruguay / Sup.: 80m² / Año: 2010
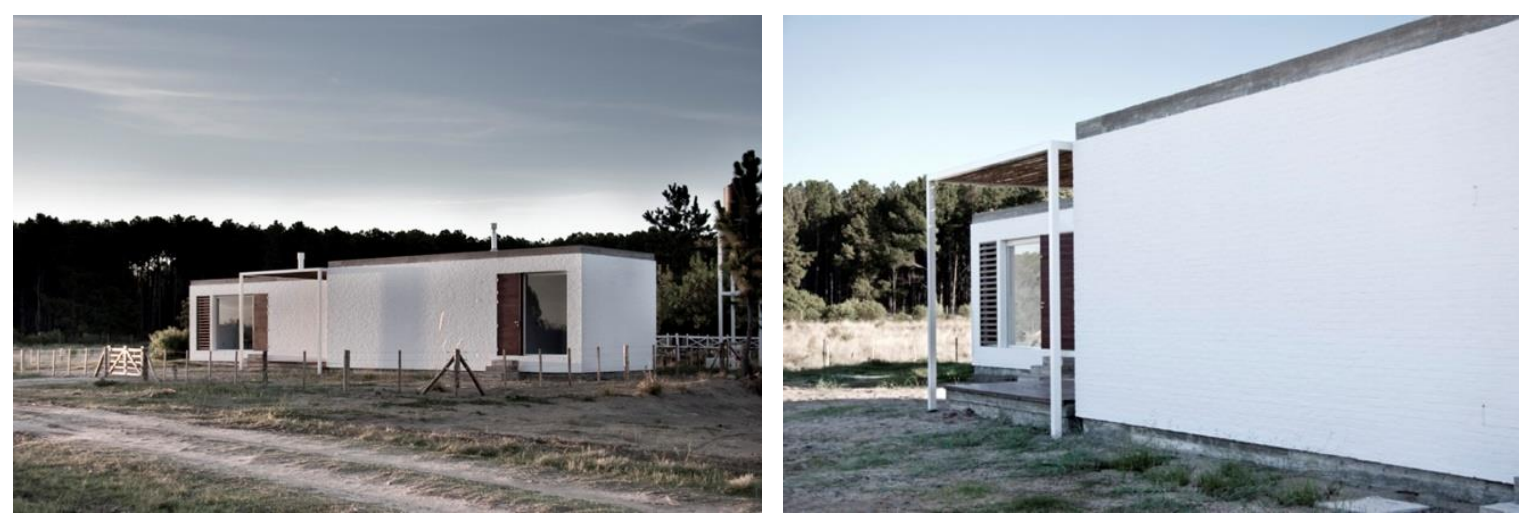

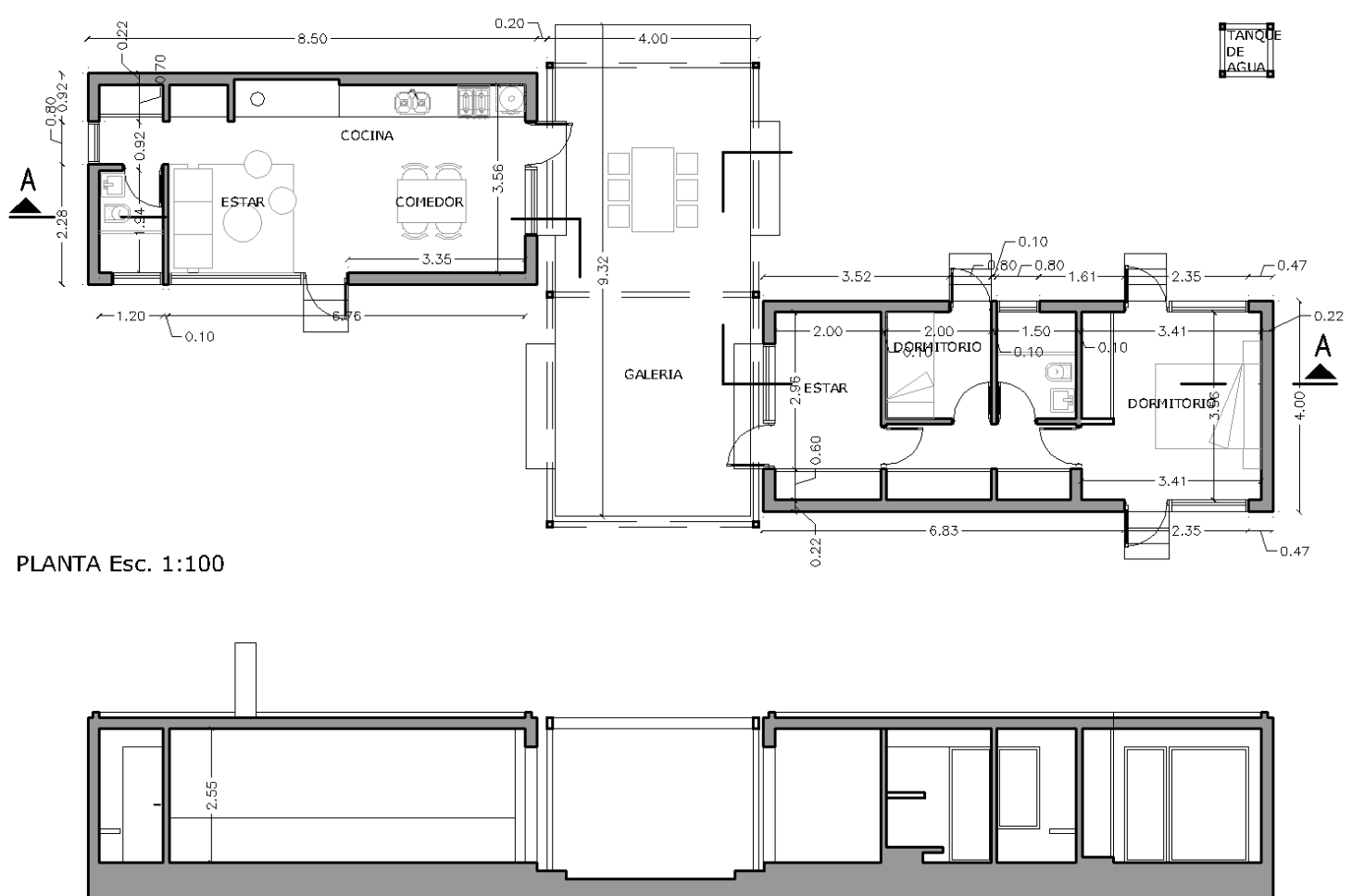

CORTE EsC. 1:100

Fig. 1: CASA ZAG / Vistas exteriores / Planta / Corte Longitudinal Fuente: https://www.plataformaarquitectura.cl/cl/762888/casa-zag-estudio-babo

Proyecto B / Casa de Bloques (Fig. 2)

Arquitectos: gualano + gualano /

Ubicación: La Pedrera, Uruguay / Sup.: 82m² / Año: 2011
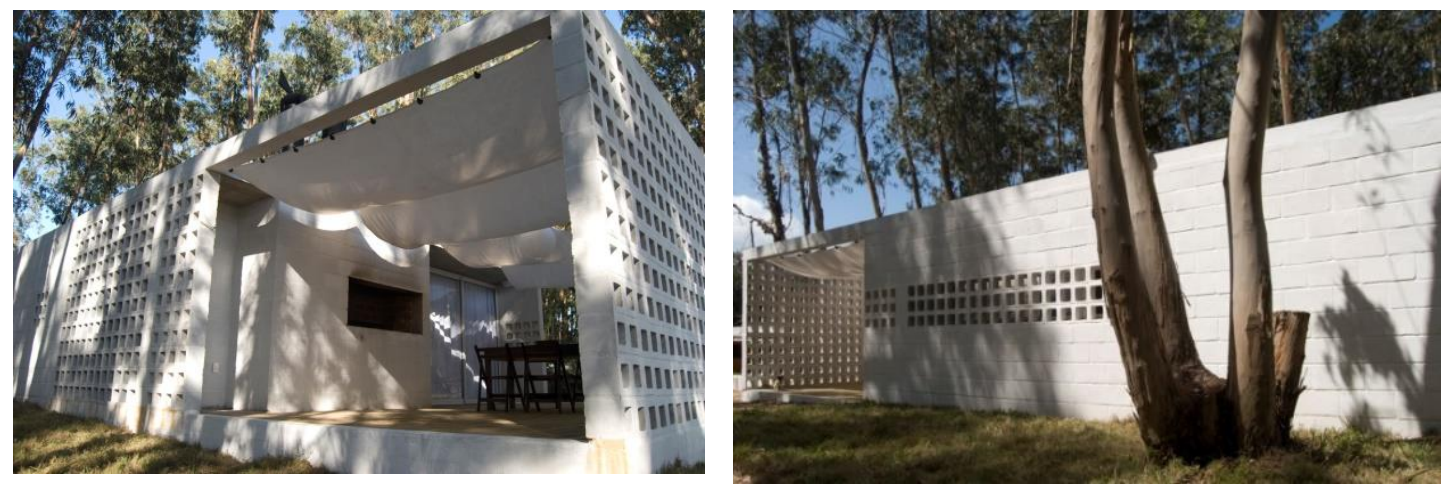


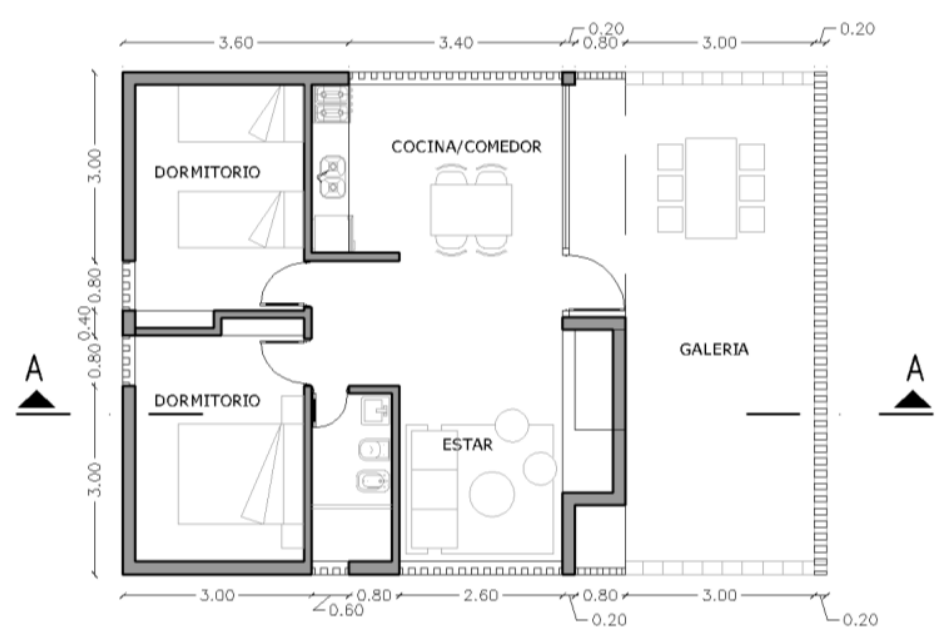

PLANTA ESC. 1:100

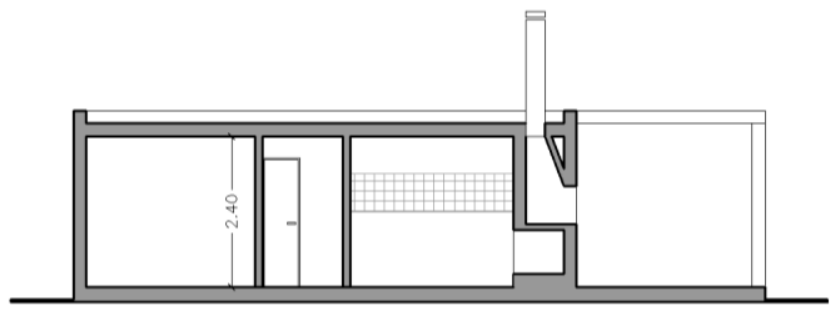

CORTE EsC. 1:100

Fig. 2: CASA DE BLOQUES / Vistas exteriores / Planta / Corte Longitudinal Fuente: https://www.plataformaarquitectura.cl/cl/02-79573/casa-de-bloques-la-pedrera-g-gualano-arquitectos

\section{Proyecto C / Casa Bio (Fig. 3)}

Arquitectos: gualano + gualano / Ubicación: Canelones, Uruguay / Sup.: 70m² / Año: 2017
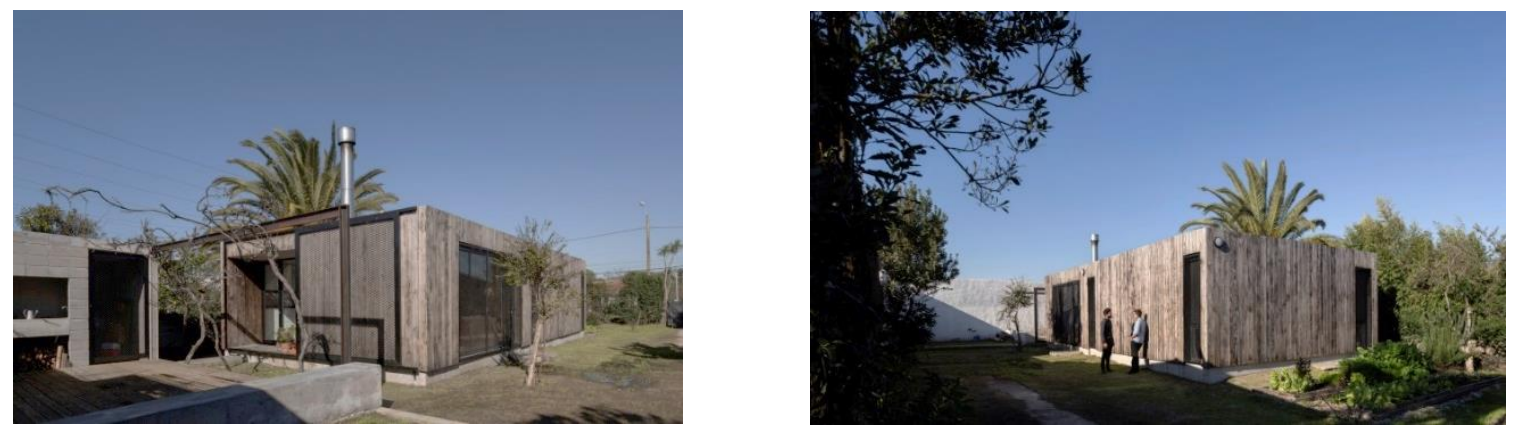

Fig. 3: CASA BIO - Vistas exteriores / Planta / Corte Longitudinal Fuentehttps://www.plataformaarquitectura.cl/cl/913424/casa-bio-gualano-plus-gualano-arquitectos 
Gustavo González; Gabriela Asís Ferri; Julieta Mansilla

Sabiendo que, los alumnos de arquitectura, tienen competencias gráficas e informáticas, habilidades para ubicarse en el espacio, facilidad para interpretar esquemas y especial interés por las imágenes que relacionan con procesos creativos y constructivos se trabaja con una metodología que incluye la utilización de recursos informáticos que contemplan las características de los estudiantes. Es por lo dicho, que este año, se introdujo la modelación tridimensional de las construcciones de mampostería.

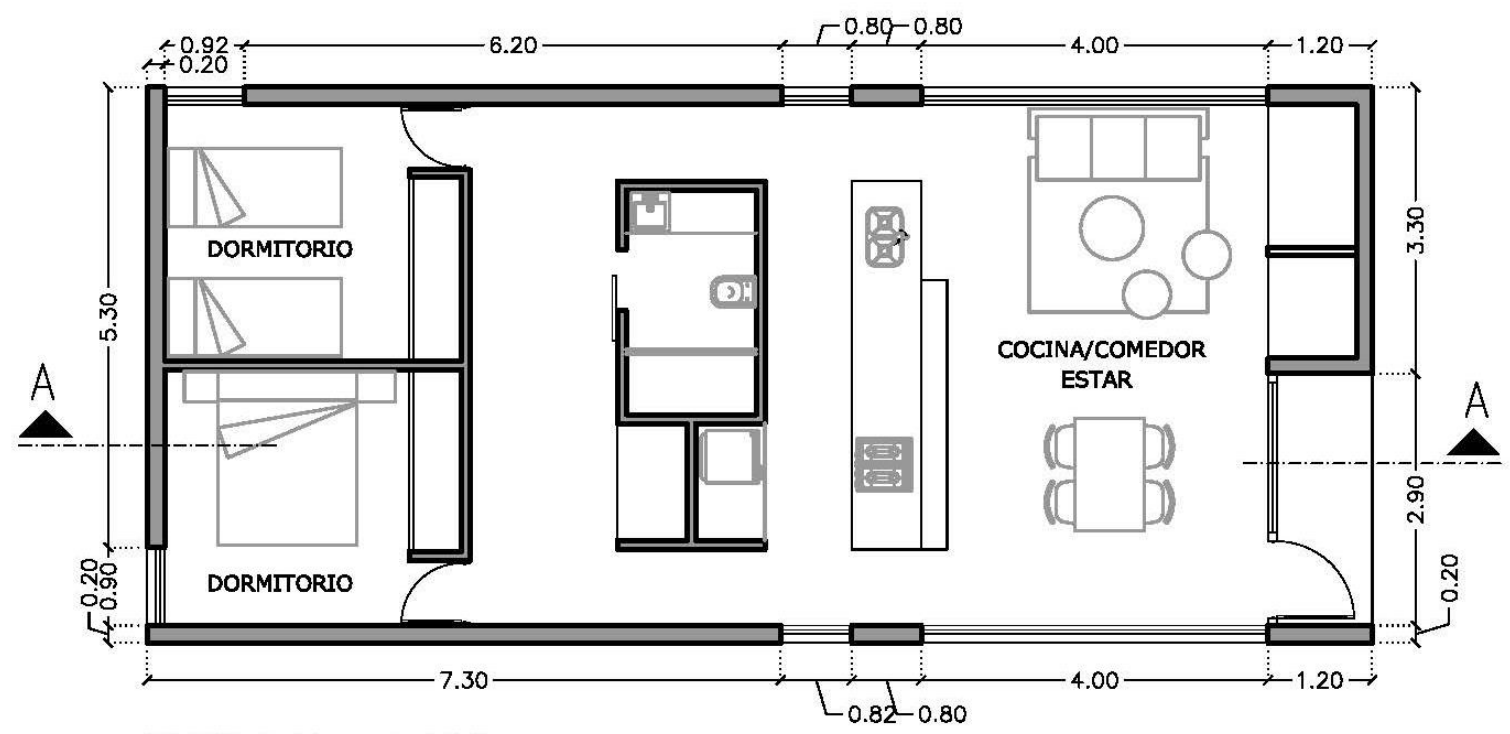

PLANTA Esc. 1:100

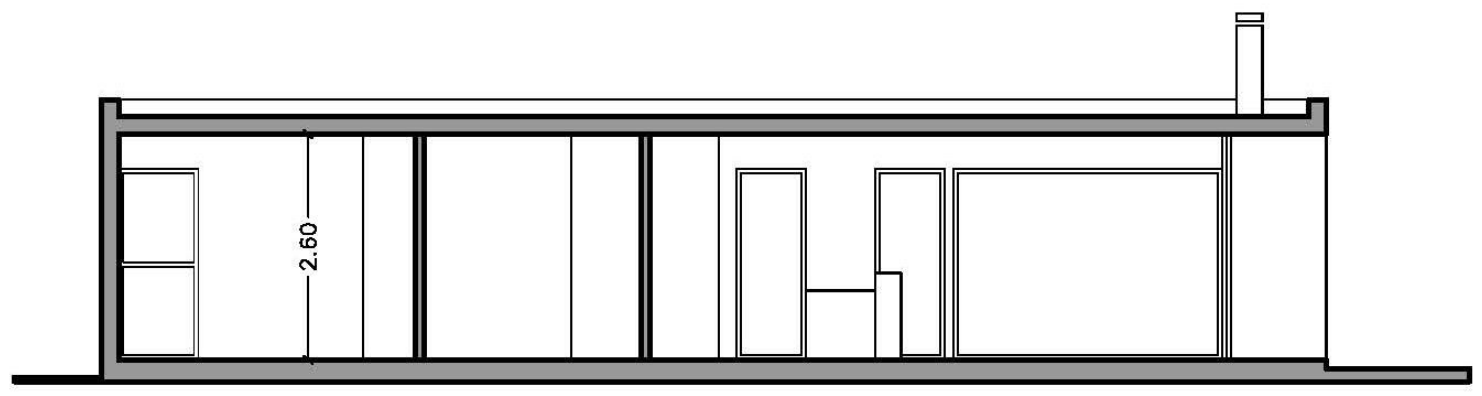

CORTE Esc. 1:100

Los modelos 3D, planteados con software adecuados, permiten al alumno visualizar, comprender y verificar conceptos más abstractos como la distorsión y la irregularidad en planta.

En la etapa de análisis se procuró que los integrantes de cada grupo interpreten los desplazamientos producidos en las construcciones por acciones sísmicas y su correlación con la regularidad en planta y la distorsión del nivel, conceptos brindados y explicados en las clases teóricas. 
Gustavo González; Gabriela Asís Ferri; Julieta Mansilla

\section{a. Proceso de Optimización}

Los alumnos evalúan su propuesta original y en función del comportamiento del mecanismo estructural frente a las acciones sísmicas, proponen distintas alternativas mejorando el comportamiento torsional y evaluando la rigidez del conjunto.

Se muestran trabajos síntesis del proceso realizado por los alumnos de los distintos ejemplos propuestos:

\section{Proyecto A}

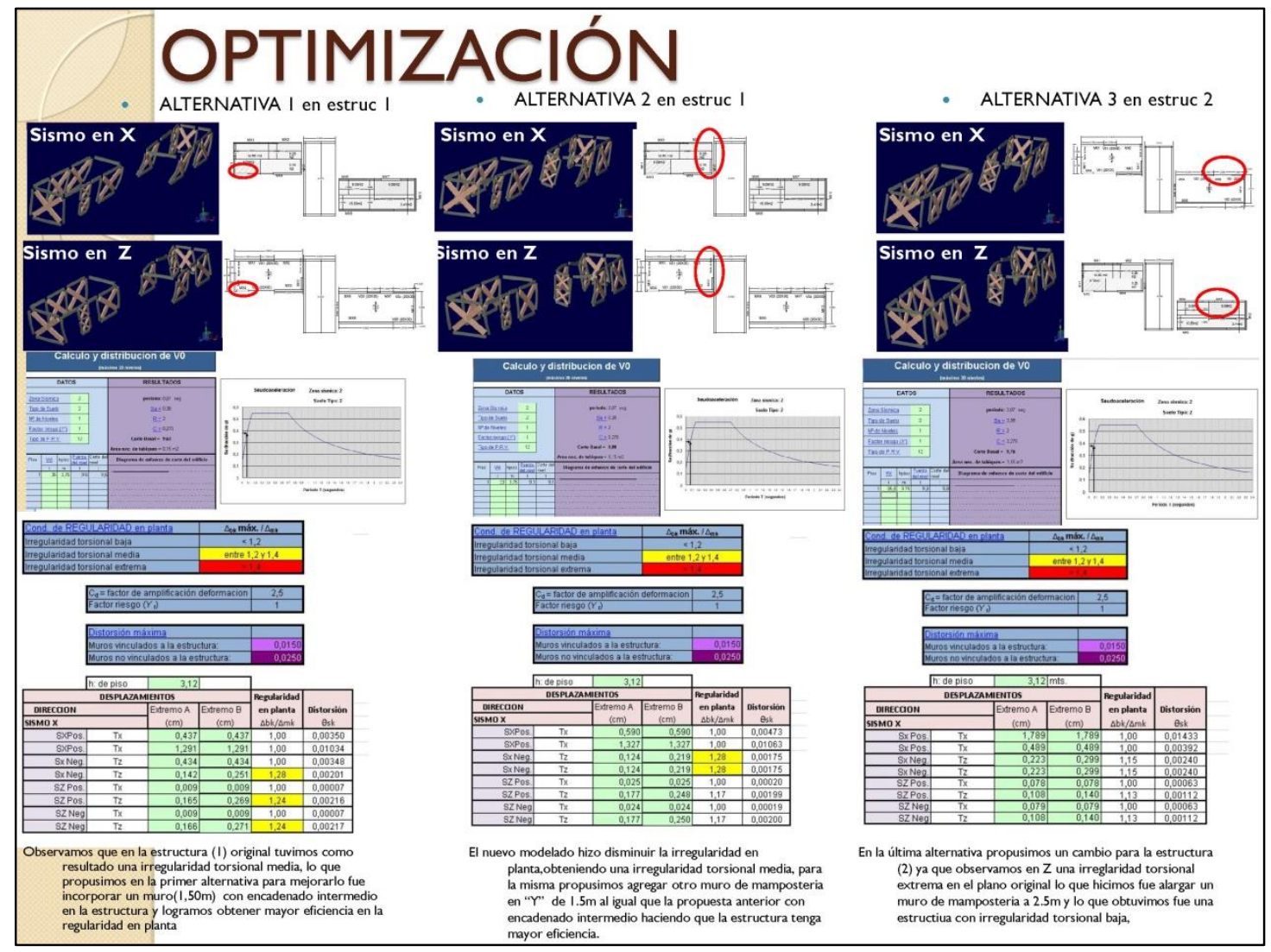

Fig. 4: CASA ZAG - Proceso de Optimización - Alumnos: Cuadrado / Nicolier / Ybañez

\section{Proyecto B}




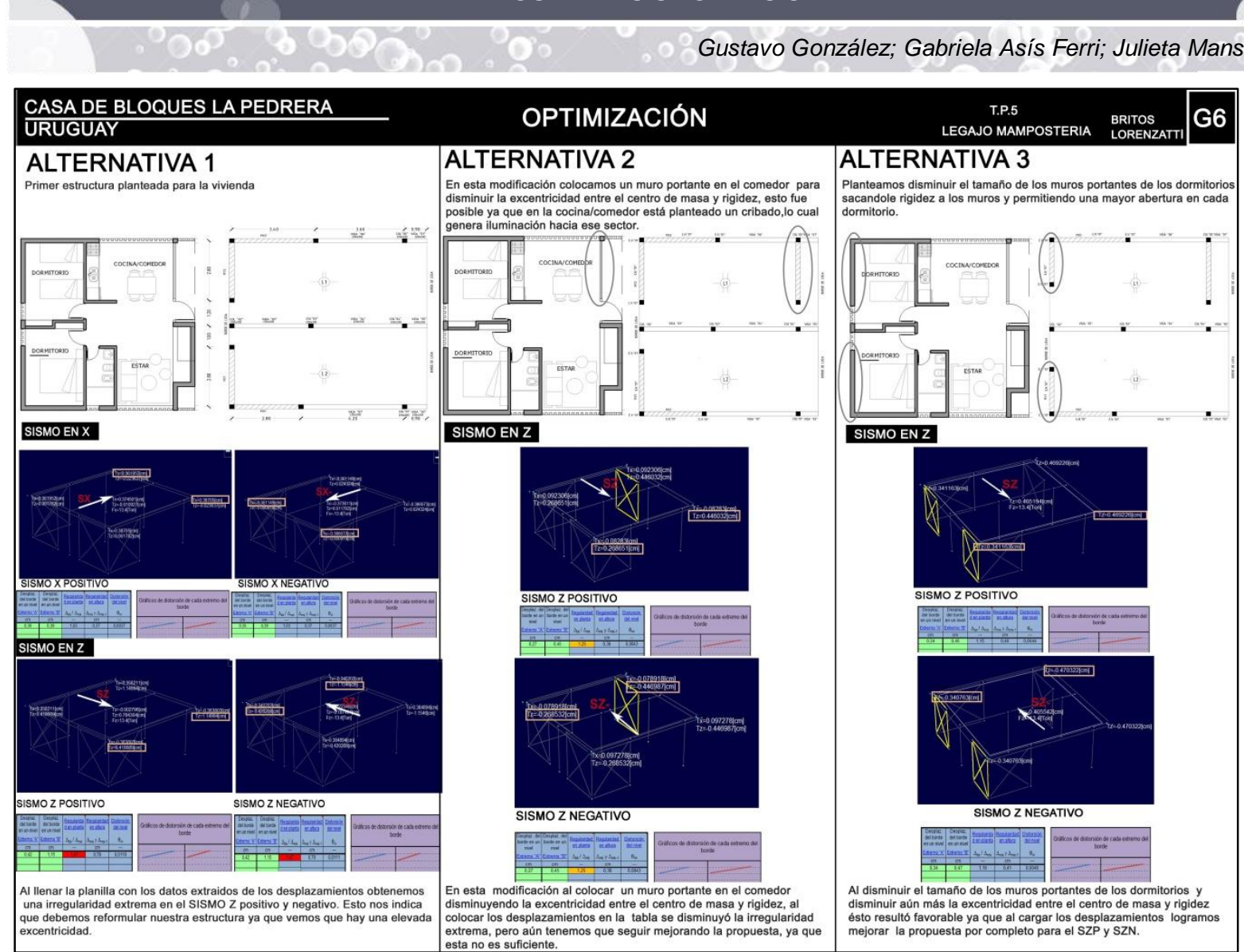

Fig. 5: CASA DE BLOQUES - Proceso de Optimización - Alumnos: Britos / Lorenzatti

A partir de una planilla Excel, elaborada por la cátedra, respetando los requerimientos reglamentarios, los estudiantes, "leen" desplazamientos que brinda el software y cuantifican las deformaciones que sufre la construcción.

En las propuestas originales que no cumplían con las demandas normativas, se plantearon alternativas estructurales coherentes y compatibles con el diseño estructural, donde se ponían de manifiesto conceptos asociados a la rigidez y a la posición de distintos elementos resistentes.

Con ajustes paulatinos, que permiten obtener conclusiones parciales, se logra encuadrar el mecanismo estructural dentro de los valores límites de regularidad en planta y altura establecidos en el INPRES-CIRSOC 103 y por lo tanto la reducción de efectos torsionales nocivos en las construcciones analizadas.

A pesar que no existe el concepto de estructura óptima o ideal, se promueve que la estructura debe ser coherente con las intenciones de diseño que se han planteado. En las decisiones estructurales y tecnológicas se encuentra presente su relación y compatibilidad con la arquitectura de la vivienda, al igual que su posibilidad constructiva.

Por otro lado, se justifica la necesidad optimizar un mecanismo propuesto a partir de lo dicho por el Arq. Daniel Moisset de Espanés: "Los recursos para construir cualquier obra son siempre limitados y también costosos. Es natural, entonces, que se procure alcanzar el máximo de objetivos con el mínimo de recursos". 
.

Gustavo González; Gabriela Asís Ferri; Julieta Mansilla

\section{Proyecto C}

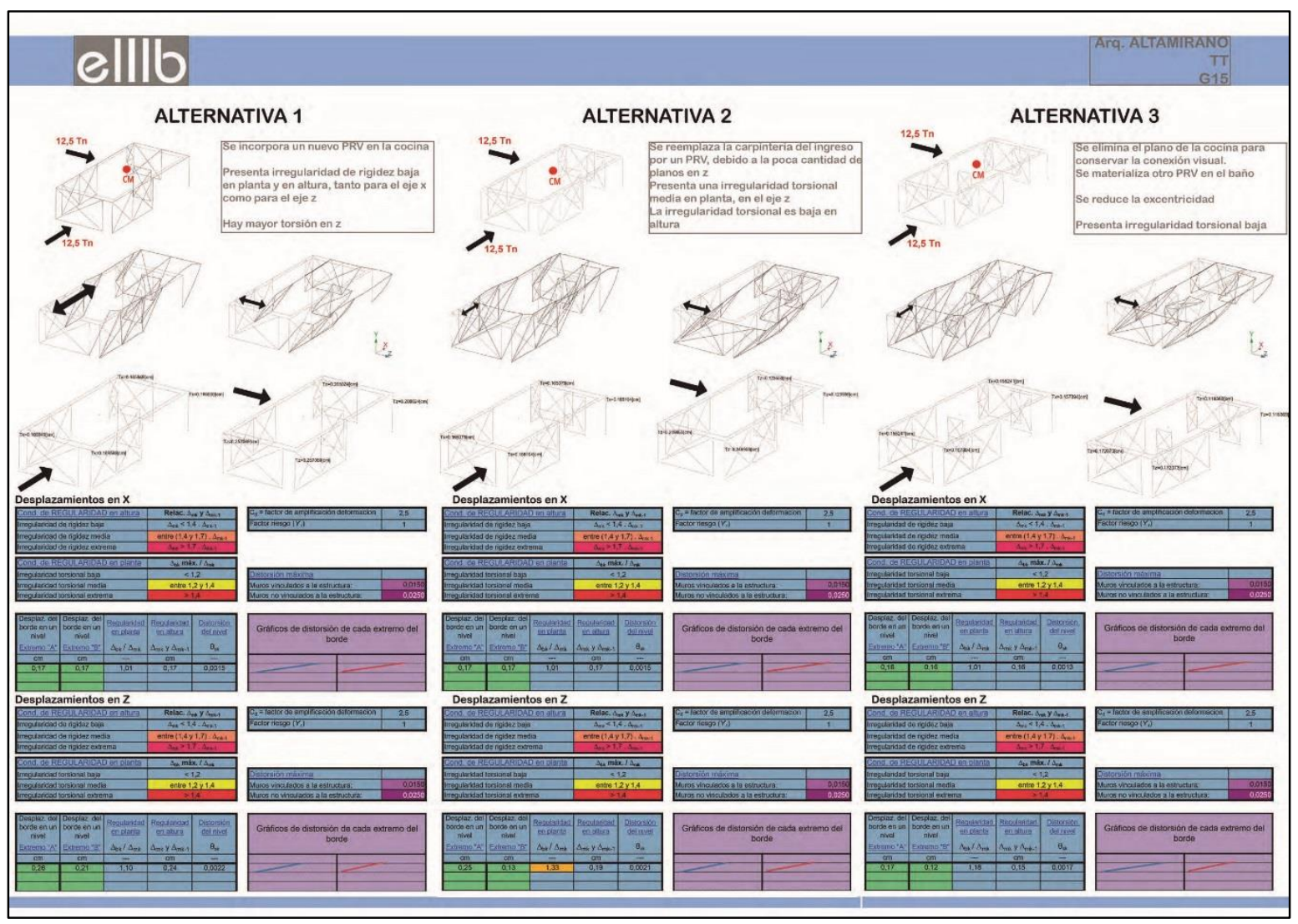

Fig. 6: CASA BIO - Proceso de Optimización - Alumnos: Blanch / Fernández / Rosales / Simón / Villena

\section{b. Verificación de Resistencia y Dimensionado de Encadenados}

La verificación de las resistencias (corte y compresión), se realizaron de acuerdo a los lineamientos de los reglamentos vigentes. Fueron programadas en una planilla de cálculo, donde se introduce las variables como dimensiones y material para obtener resistencias y comparar con las demandas sísmicas y gravitatorias.

El mismo recurso se utilizó para obtener las armaduras longitudinales y estribos de los encadenados verticales y horizontales de cada muro. En este punto se hizo especial énfasis en la importancia del anclaje de las barras longitudinales y las separaciones de estribos en las zonas que marca el reglamento, promoviendo el concepto que el mecanismo estructural se comporta como se construye y no como se calcula.

Se muestran algunos detalles que se elaboran a partir de la interpretación del comportamiento del muro y los resultados que se obtienen de las planillas. (Fig. 7) 
10630 Gustavo González; Gabriela Asís Ferri; Julieta Mansilla

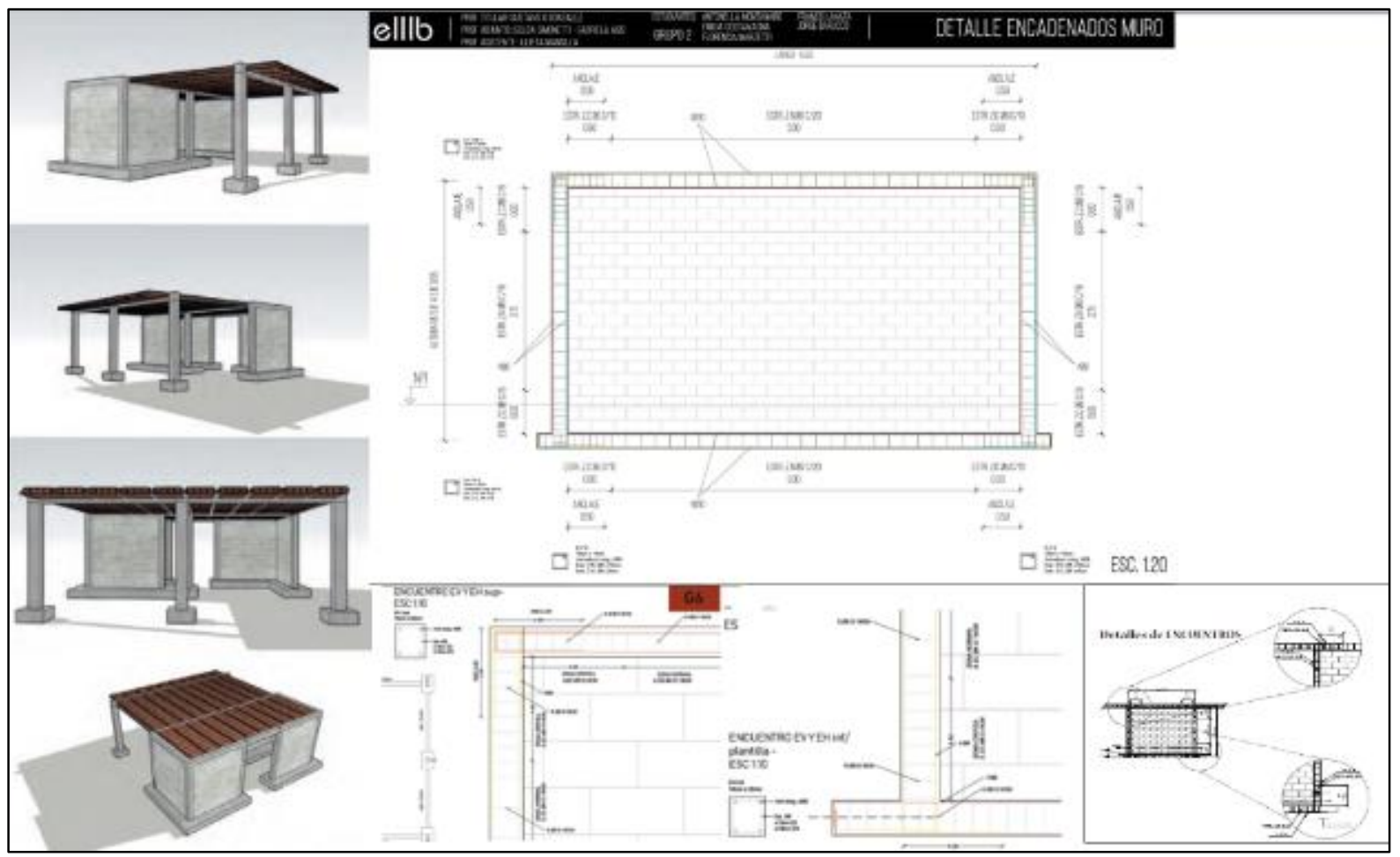

Fig. 7: CASA E BLOQUES - Dimensionado de encadenados - Detallado - Alumnos: Collura / Helmbrecht / Juarez Silvetti / Mellado / Villagran.

A modo de cierre del ejercicio, los estudiantes elaboran una maqueta en escala 1:10. La clase práctica de armado y unión de encadenados de un panel, colabora en gran medida a justificar la importancia de los detalles de: doblado, anclaje, zonas críticas de estribos y su relación con los esfuerzos internos que se producen en un muro de mampostería con encadenados simple. (Fig. 8)
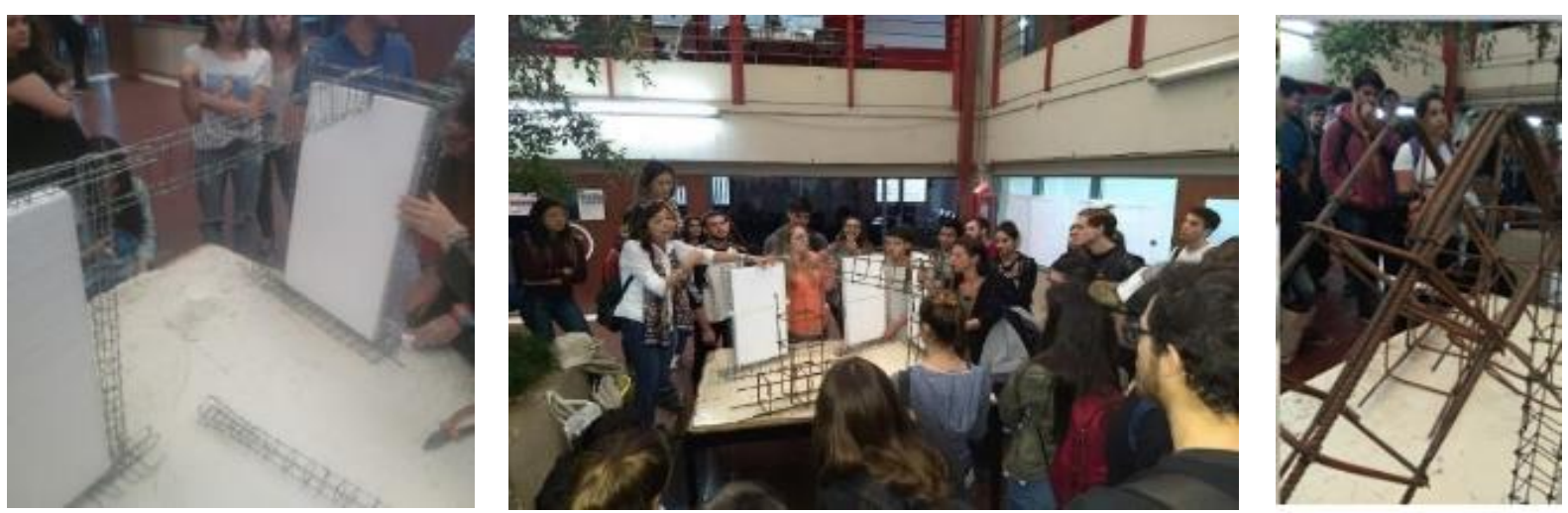

Fig. 8: Elaboración de detalles - Trabajo de alumnos - Taller de maquetas 
Gustavo González; Gabriela Asís Ferri; Julieta Mansilla

En la última clase destinada a este práctico, los alumnos exponen sus trabajos de manera digital, mostrando de qué manera hicieron más eficientes las propuestas presentadas e intercambiando distintas soluciones.

De esta forma, visualizan que el mecanismo estructural se diseña, por lo tanto no existe una única respuesta al "ejercicio planteado".

\section{CONCLUSIONES}

La concepción metodológica de la Cátedra implica considerar que la estructura en primera instancia, se diseña, luego se analiza y por último se verifica. Atendiendo a este concepto, resulta sumamente importante que el alumno pueda interpretar y analizar cualitativamente, para luego realizar verificaciones cuantitativas simples y mejorar su comportamiento.

Una pequeña obra de arquitectura es el pretexto para abordar una comprensión a la vez que integral, más concreta del mecanismo estructural. Plantear en ella ese mecanismo estructural y generar su aptitud tanto para acciones horizontales como para cargas gravitatorias, implica un doble reconocimiento; en primer lugar de los planos verticales aptos para responder a solicitaciones de naturaleza sísmica y de sus posibles optimizaciones para mejorar la eficiencia del mecanismo en ese sentido, y en segundo lugar la respuesta del conjunto frente a las ineludibles acciones gravitatorias

Las herramientas informáticas, adecuadamente implementadas, estimulan y apoyan la tarea de los estudiantes. La representación del mecanismo estructural en 3D, ayuda a visualizar, el comportamiento de los planos resistentes verticales frente a las acciones a los que están sometidos y optimizar su comportamiento frente a cargas de distinta naturaleza.

La construcción de modelos en esc 1:10 de planos resistentes verticales enfatiza la necesidad de una correcta materialización de los distintos componentes de un objeto de arquitectura.

Finalmente, la última intención, es la generar, en el estudiante de los últimos años, habilidades para la integración de los conocimientos al conjunto de conceptos y contenidos vistos en los cursos anteriores, fomentando una actitud reflexiva para que desarrolle su propio juicio crítico, su capacidad de investigación y orientarlo hacia la aplicación profesional.

\section{BIBLIOGRAFÍA}

Saleme, Horacio., Comoglio, S., \& Muñoz, M. (2000). Tucumán: Educación y Sismo. Revista de Ciencias Exactas E Ingeniería - U.N.T. - Numero 18.

Reboredo, Agustín (2017). El Diseño Estructural. Mendoza. Editorial Nobuko.

INPRES - CIRSOC. (2018). Reglamento argentino para construcciones sismorresistentes - parte I Construcciones en General.

INPRES - CIRSOC. (2018). Reglamento argentino para construcciones sismorresistentes - parte III Construcciones de Mampostería. 